\title{
Energy Cooperation Optimization in Residential Microgrid with Virtual Storage Technology
}

\author{
Jun Tang, ${ }^{1}$ Chen Yang, ${ }^{1}$ Changsen Feng $\mathbb{D}^{2}{ }^{2}$ Junting Li, ${ }^{1}$ Xiaowen Gu, ${ }^{1}$ and Xuelei Jiang ${ }^{1}$ \\ ${ }^{1}$ State Grid Suzhou Power Supply Company, Suzhou 215004, China \\ ${ }^{2}$ College of Information Engineering, Zhejiang University of Technology, Hangzhou 310023, China
}

Correspondence should be addressed to Changsen Feng; fcs@zjut.edu.cn

Received 25 September 2020; Revised 15 October 2020; Accepted 19 January 2021; Published 29 January 2021

Academic Editor: Omar Jacobo Santos Sanchez

Copyright $\left({ }_{0} 2021\right.$ Jun Tang et al. This is an open access article distributed under the Creative Commons Attribution License, which permits unrestricted use, distribution, and reproduction in any medium, provided the original work is properly cited.

The power balance of the tie-line is crucial to the stable operation of a community microgrid. This paper presents a power fluctuation smoothing method of the microgrid tie-line based on virtual energy storage technology. Firstly, the structure characteristics and the energy coupling mode of the combined heat and power system is systematically analyzed. Considering the operating characteristics of heat pumps, micro gas turbines, and buildings' heat storage characteristics, a virtual energy storage model is established. Secondly, the target power of the tie-line is determined with the storage state indexes into consideration. Subsequently, a power allocation strategy which takes into account the correction of equipment state mapping set is proposed to allocate the tie-line power fluctuations to heat pumps, micro gas turbines, and supercapacitors. Simulation results show this method can realize the coupling coordination between heat and power energy and ensure the smoothing effect of the power fluctuations. Meanwhile, the control flexibility of the combined heat and power system can be enhanced, and the microgrid's operating economy can be improved.

\section{Introduction}

Along with the popularization and application of renewable energy, the distributed power supply system composed of wind power and photovoltaic can form a user-oriented community microgrid [1-3]. During the past decade, the combined heat and power (CHP) system has gained increasing concerns and is regarded as the key of the community microgrid to meet users' multiple demands for electricity and heat $[4,5]$. Renewable energy and traditional energy are collaboratively utilized to provide system optimization management and regulation in the community microgrid [6-8]. In the traditional mode, the community microgrid generally realizes the power balance of the tie-line through the configuration of battery energy storage to ensure the security operation of the system, but this will increase the operation cost due to the high price of the battery energy storage. Coordinated control of heat and power energy can smooth the tie-line's power fluctuations and realize multienergy supply and cascade utilization, and therefore the overall investment costs of the microgrid can be reduced [9-11].

At present, battery energy storage system (BESS) $[12,13]$ and supercapacitors $[14,15]$ are widely utilized in the energy cooperation optimization [16-18]. In [19], the optimal performance function is presented to obtain the control sequence of the BESS, while with the total electricity cost minimized and the battery's lifetime simultaneously extended. Hierarchical control, composed of centralized and distributed control mode, is proposed in [20] to minimize bus voltage deviation and maximize the utilization of the hybrid energy storage system's utilization capacities, respectively. Although the power fluctuations could be effectively smoothed by the BESS, its popularity is still limited by the high investment cost [21,22].

Heat pumps, micro gas turbines, and other heating equipment can achieve coordinated control of heat and power energy through electric-thermal conversion and CHP technology, while with the power fluctuations of the tie-line smoothed and the thermal demand of the microgrid satisfied 
$[23,24]$. In [25], switching of switches state of heat pumps can effectively reduce the numbers of energy storage charge and discharge conversion and smooth power fluctuations of the microgrid tie-line. An electrothermal joint model, which aims at reducing the power fluctuations of the tie-line and the operating cost of the microgrid, is established in [26]. However, this condition does not consider the impact of heat production on user comfort. The above research mainly focuses on single heating equipment, and the deep coupling of heat and power energy demand further investigation. In order to furtherly explore the advantages of energy complementarity and collaborative control of the CHP system, it is necessary to comprehensively consider various types of heating equipment, and make full use of the flexibility of heat energy regulation to smooth the power fluctuations of the tie-line.

In the CHP system, the microgrid energy control centre increases/reduces the heat power to regulate the electric power of the heat pumps and the power output of the micro gas turbines, thus undertaking the smoothing power fluctuations of the tie-line. Due to the heat insulation effect of building walls, the response speed of indoor temperature to electric power changes is relatively slow. Thus, its heat storage characteristics can be compared to the charging/discharging characteristic of energy storage $[27,28]$.

Given this background, this paper is devoted to addressing the power fluctuations of microgrid tie-line considering the virtual energy storage. The structure of the community microgrid is introduced and virtual storage is modeled considering the characteristics of the CHP system and the heat storage characteristics of buildings. Also, the allocation strategy of power fluctuations of microgrid tieline is presented to determine the output power of micro gas turbines, heat pumps, and supercapacitors, and therefore the power fluctuations smoothing can be realized and the comprehensive utilization efficiency of energy can be improved. The contributions could be summarized as follows:

The energy coupling mode of the CHP microgrid is analyzed.

The operating characteristics of heat pumps, micro gas turbines, and heat storage characteristics of buildings are elaborately analyzed, and then a virtual energy storage model is established.

A strategy for smoothing community microgrid tie-line power considering the virtual storage technology is presented.

A correction factor is designed to determine the final output power based on the equipment state mapping set.

The remainder of this paper is given as follows. Section 2 proposed the structure of the community microgrid, including the microelectricity network and microthermal network. In Section 3, the heat pumps, micro gas turbines, supercapacitor, and virtual energy storage are modeled. The tie-line's target power is determined in Section 4, and the allocation strategy of the target power of the tie-line is detailed in Section 5. The simulation results of the presented model are described in Section 6. The final section gives the conclusions.

\section{System Structure of Community Microgrid}

The community microgrid structure is depicted in Figure 1, which includes the microelectricity network and microthermal network. Distributed generation such as wind power and photovoltaic generate and input electric energy into the microelectricity grid, and supercapacitors are used to smooth the power fluctuations of the tie-line of the microgrid. The micro gas turbines input electric energy to the microelectricity network by burning natural gas and recycle the waste thermal energy through the absorption heat engine to generate heat and input it to the microthermal network, so as to realize the cogeneration of heat and power. Based on electric-thermal conversion technology, the heat pumps can convert low-quality thermal energy in the environment into high-quality thermal energy, in which part of electric energy is consumed by the heat exchanger and compressor and thermal energy are output to microthermal network. The electric energy and thermal energy are finally transmitted to the community to meet the demand for electric power and thermal energy on the user side. The power flow and heat flow are marked with green and red in Figure 1, respectively.

$$
\begin{aligned}
P_{\text {tie-line }}(t)= & P_{\mathrm{EL}}(t)+P_{\mathrm{HP}}(t)+P_{\mathrm{SC}}(t) \\
& -P_{\text {wind }}(t)-P_{\mathrm{PV}}(t)-P_{\mathrm{MT}}(t) .
\end{aligned}
$$

The electric power balance equation of community microgrid at time $t$ is described in equation (1), where $P_{\text {tie-line }}(t)$ is defined as real-time power of tie-line at time $t$, and the value of energy flowing into the microgrid is positive and negative otherwise. $P_{\mathrm{sc}}(t)$ represents the charging/discharging power of the supercapacitors at time $t$ and is positive with charging and negative with discharging. $P_{\text {wind }}(t)$ and $P_{\mathrm{pv}}(t)$ denote the output of wind power and photovoltaic at time $t$, respectively. $P_{\mathrm{MT}}(t)$ and $P_{\mathrm{HP}}(t)$ represent the power of micro gas turbines and heat pumps at time $t$, respectively. $P_{\mathrm{EL}}(t)$ represents the load demand of the community at time $t$ :

$$
Q_{\mathrm{TL}}(t)=Q_{\mathrm{HP}}(t)+Q_{\mathrm{MT}}(t) .
$$

The heat power balance equation is described in equation (2), where $Q_{\mathrm{TL}}(t)$ represents heat demand power of users at time $t$ in the microgrid and $Q_{\mathrm{HP}}(t)$ and $Q_{\mathrm{MT}}(t)$ denote the heat power of heat pumps and micro gas turbines at time $t$, respectively.

\section{Modeling of Virtual Energy Storage}

The air conditioning load is assumed as the heat demand in the microgrid. Since the user's demand for temperature is adjustable, the heat pumps and micro gas turbines can change heat demand to participate in the power smoothing of tie-line, which is similar to the charging/discharging characteristics of the energy storage system. Therefore, heat pumps and micro gas turbines can form a virtual storage system. The output can be adjusted to smooth power fluctuations of tie-line and ensure that the room temperature is reasonable. 


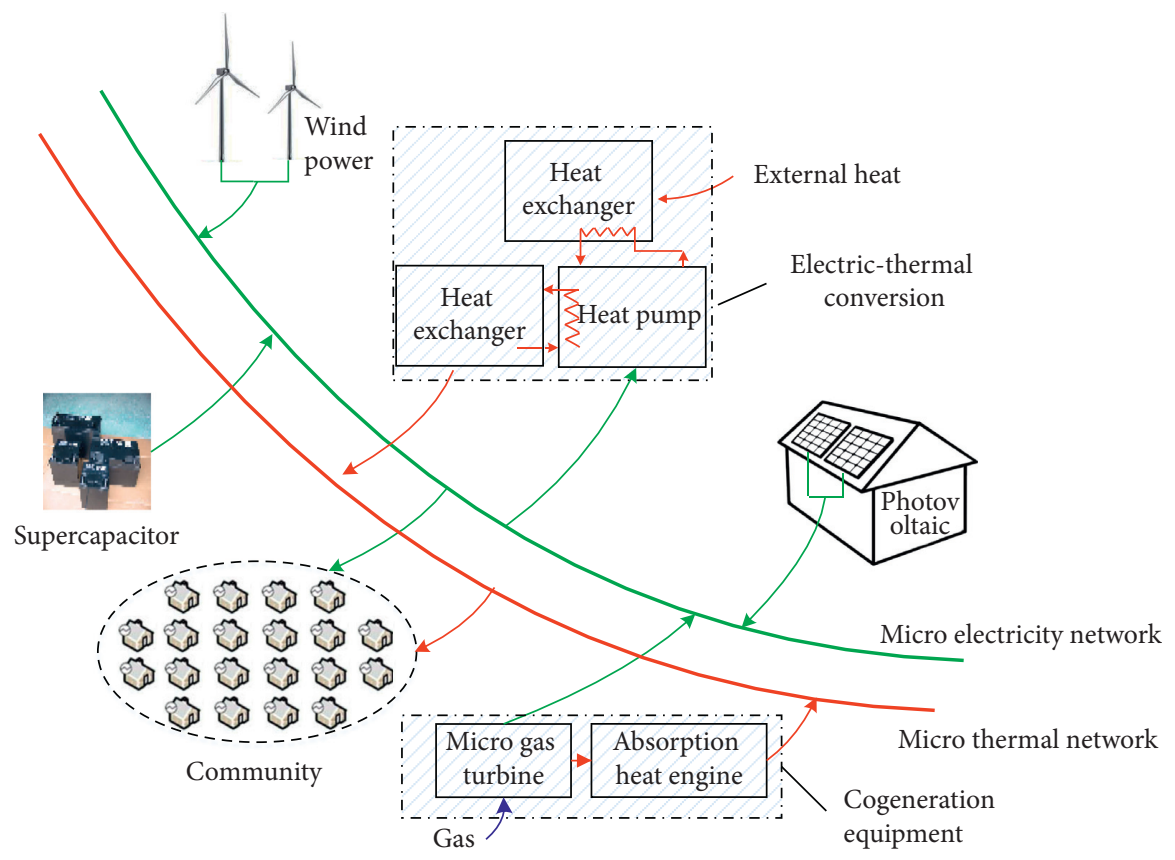

FIGURE 1: Energy flow in community microgrid combined with heat and power system.

3.1. Micro Gas Turbine. Under the condition of CHP cogeneration, the electric power and heat power of the micro gas turbine are shown as follows:

$$
\begin{aligned}
P_{\mathrm{MT}}(t) & =P_{\mathrm{gas}}(t) \times \eta_{\mathrm{MT}}, \\
\mathrm{Q}_{\mathrm{MT}}(t) & =\gamma_{\mathrm{MT}} \times \eta_{\text {heat }} \times P_{\mathrm{MT}}(t) \times \mathrm{COP}_{\mathrm{MT}},
\end{aligned}
$$

where $P_{\mathrm{MT}}(t)$ and $Q_{\mathrm{MT}}(t)$ represent the electric power and heat power of the micro gas turbine at time $t$, respectively; $P_{\text {gas }}(t)$ denotes the gas power injected into the micro gas turbine at time $t ; \eta_{\mathrm{MT}}$ represents the power generation efficiency of the micro gas turbine; $\gamma_{\mathrm{MT}}$ and $\eta_{\text {heat }}$ represent the cogeneration ratio and heat transfer efficiency of the micro gas turbine; and, $C O P_{\mathrm{MT}}$ is the heating energy efficiency coefficient of the micro gas turbine.

3.2. Heat Pump. The heat pump can convert low-grade heat energy into high-grade heat energy by consuming a small amount of electric energy. The real-time power consumption of the heat pump changes correspondingly with the working fluid flow:

$$
\begin{aligned}
& \operatorname{COP}_{\mathrm{HP}}=\frac{Q_{\mathrm{HP}}}{P_{\mathrm{HP}}}, \\
& \mathrm{Q}_{\mathrm{HP}}(t)=C_{\mathrm{HP}} \times \rho_{\mathrm{HP}} \times v(t) \times \Delta T_{\mathrm{HP}},
\end{aligned}
$$

where $\mathrm{COP}_{\mathrm{HP}}$ is the heating energy efficiency coefficient of heat pump, $C_{\mathrm{HP}}$ and $\rho_{\mathrm{HP}}$ represent the specific heat and density of heat pump working fluid, $v(t)$ denotes the flow of heat pump working fluid at time $t$, and $\Delta T_{\mathrm{HP}}$ is the temperature difference before and after one working cycle.

3.3. Super-capacitor. The state of charge (SOC) of the supercapacitors can be described as follows:

$$
\begin{gathered}
\operatorname{SOC}_{\mathrm{SC}}(t)=\operatorname{SOC}_{\mathrm{SC}}(t-1)+\frac{P_{\mathrm{SC}}(t)}{E_{\mathrm{SC}}}, \\
\operatorname{SOC}_{\mathrm{SC}_{-} \min } \leq \operatorname{SOC}_{\mathrm{SC}}(t) \leq \mathrm{SOC}_{\mathrm{SC} \_ \text {max }},
\end{gathered}
$$

where $\mathrm{SOC}_{\mathrm{SC}}(t)$ represents the SOC of supercapacitors at time $t, E_{\mathrm{SC}}$ is the capacity of the supercapacitors, and $\mathrm{SOC}_{\mathrm{SC}_{-} \max }$ and $\mathrm{SOC}_{\mathrm{SC}_{-} \text {min }}$ are the upper limit and lower limit of the SOC of supercapacitors, respectively.

3.4. Virtual Energy Storage. Based on the laws of thermodynamics $[29,30]$, the heat balance equation of the virtual energy storage system can be described as

$$
\frac{\mathrm{d} Q}{\mathrm{~d} t}=\rho \times C \times V \times \frac{\mathrm{d} T_{\text {inside }}}{\mathrm{d} t},
$$

where $Q$ represents the indoor heat, $\rho$ and $C$ are the air density and specific heat capacity of air, respectively; and $V$ and $T_{\text {inside }}$ represent the indoor air capacity and the indoor temperature, respectively.

Heat pumps and micro gas turbines provide heat to users, and the indoor heat is also related to heat transfer and heat radiation caused by indoor and outdoor temperature differences. Equation (6) can be furtherly expressed as 


$$
\begin{aligned}
\rho \times & \times V \times \frac{\mathrm{d} T_{\text {inside }}}{\mathrm{d} t} \\
= & Q_{\mathrm{HP}}(t)+Q_{\mathrm{MT}}(t)+(t) \times S_{\text {window }} \times k_{S} \\
& +\left(k_{\text {wall }} \times S_{\text {wall }}+k_{\text {window }} \times S_{\text {window }}\right) \\
& \times\left[T_{\text {outside }}(t)-T_{\text {inside }}(t)\right]
\end{aligned}
$$

where $k_{\text {wall }}$ and $k_{\text {window }}$ are heat transfer coefficients of wall and window [29], respectively; $S_{\text {wall }}$ and $S_{\text {window }}$ are the area of wall and window, respectively; $I(t)$ represents the solar radiation power at time $t ; k_{\mathrm{s}}$ is the radiation coefficient, which is related to the glass material [1].

Suppose only a single type of heating equipment (e.g., heat pump) is applied to participate in smoothing power fluctuations. In that case, the tie-line's instantaneous power change will directly affect the heating equipment's heat power, causing the room temperature's instability on the user side. This control mode is accessible, but the control effect is not satisfying. Nevertheless, the cogeneration system in this paper includes two types of equipment, heat pumps and micro gas turbines. When the power fluctuations of the tie-line are positive, the heat pumps can undertake parts of the fluctuations component by increasing the compressor's power, and the rise of heat production causes the increase in room temperature on the user side. Simultaneously, the micro gas turbines can bear another part of power fluctuations by reducing natural gas injection power, and the drop in heat production can cause room temperature reduction (the analysis of tie-line power fluctuation being negative is similar). Through coordinated control of heat pumps and micro gas turbines, the range of room temperature variation on the user side is smaller and the effect of temperature control can be greatly enhanced. As a result, compared with a single type of heating equipment, the virtual energy storage composed of heat pumps and micro gas turbines has higher control flexibility and heating stability.

\section{Determination of Target Power of Microgrid Tie-Line}

The microgrid energy control centre comprehensively considers the real-time power of renewable energy, user load, and the state of supercapacitors and virtual energy storage and then determines the target power of the tie-line.

State indexes of virtual energy storage and supercapacitors are introduced to measure the ability of smoothing fluctuating power of the tie-line:

$$
\begin{gathered}
S_{\mathrm{T}}(t)=\frac{\left[T_{\text {inside }}(t)-T_{\text {inside0 }}\right] \times 2}{T_{\text {inside_max }}-T_{\text {inside_min }}}, \\
S_{\mathrm{SC}}(t)=\frac{\left[\mathrm{SOC}_{\mathrm{SC}}(t)-\mathrm{SOC}_{\mathrm{SC} 0}\right] \times 2}{\mathrm{SOC}_{\mathrm{SC} \_ \text {max }}-\mathrm{SOC}_{\mathrm{SC} \_ \text {min }}},
\end{gathered}
$$

where $S_{\mathrm{T}}(t)$ and $S_{\mathrm{SC}}(t)$ represent the state indexes of virtual energy storage and supercapacitors, respectively. $T_{\text {inside-max }}$ and $T_{\text {inside-min }}$ are the upper and lower limit of room temperature; $T_{\text {inside } 0}$ is the target reference value of room temperature, and the value is specified as $\left(T_{\text {inside-max }}+T_{\text {inside-min }}\right) / 2$ in this paper. Similarly, $\mathrm{SOC}_{\mathrm{SC} 0}$ represents the target reference value of the supercapacitors' SOC and the value is set as $\left(\mathrm{SOC}_{\mathrm{SC} \_ \text {max }}+\mathrm{SOC}_{\mathrm{SC} \_ \text {min }}\right) / 2$. It can be seen that the value range of both the virtual energy storage state index and the supercapacitors state index is $[-1,1]$. When the value is close to 1 , the energy release characteristic is strong, but the energy absorption characteristic is very weak. When the value is close to -1 , it indicates that the energy absorption characteristic is robust, but the energy release characteristic is weak. When the energy storage state index is close to 0 , it means that it has strong energy charging/discharging characteristics, which can be used as an ideal index of smoothing tie-line power.

The weighted sliding average filter algorithm is used to smooth the power fluctuations and determine the tie-line's target power. When the state index of virtual energy storage or supercapacitors is good, it means the capacity of charging/ discharging is relatively strong, and the number of filtering should be increased correspondingly to improve the smoothing effect of power fluctuations. When the state index is poor, the number of filtering shall be decreased to reduce the smoothing effect of power fluctuations, so as to ensure that the virtual energy storage and supercapacitors are in an appropriate state:

$$
N(t)=\left(2-\left|S_{\text {SC }}(t-1) \times S_{T}(t-1)\right|\right) \times N_{\text {base }},
$$

where $N(t)$ is the number of sliding average filtering at time $t$ and $N_{\text {base }}$ is defined as the base value of sliding average filtering.

In order to improve the effectiveness of the sliding average filter algorithm for smoothing the power fluctuations of the tie-line, a weight factor is introduced to ensure the algorithm focuses more on the power near time $t$ and weakens the power influence away from time $t$. The expression of the weight factor can be described as follows:

$$
\beta(k)=1-\lambda \times\left(1-\frac{k}{N}\right), \quad k=1,2, \ldots, N,
$$

where $\beta(k)$ is the weight factor, $\lambda$ is the slope of the weight factor, and the larger the value is, the more obvious the effect of weight factor is, that is, more emphasis is placed on the power of tie-line in the adjacent time $t$ when filtering. In this paper, $\lambda$ is taken as 0.5 . The target power of the tie-line at time $t$ can be expressed through the sliding average filter algorithm:

$$
P_{\text {tie-line } 0}(t)=\frac{\sum_{k=1, t=t-N+1}^{N, t} \beta(k) \times P_{\text {tie-line }}(t)}{\sum_{k=1}^{N} \beta(k)},
$$

where $P_{\text {tie-line0 }}(t)$ represents the tie-line's target power at time $t$. 
The flowchart of the sliding average filtering algorithm to determine the tie-line's target power of the microgrid at time $t$ is shown in Figure 2.

\section{Allocation Strategy of Power Fluctuations of Microgrid Tie-Line}

After determining the tie-lines target power, the difference between real-time power and the tie-line's target power is the power fluctuations of tie-line, which should be smoothed by heat pumps, micro gas turbines, and supercapacitors. The power smoothing target of the microgrid tie-line at time $t$ is shown in the following equation:

$$
\begin{aligned}
P_{\text {tie-line }}(t) & -P_{\text {tie-line } 0}(t) \\
& =P_{\mathrm{SC}}(t)+P_{\mathrm{HP}}(t)-P_{\mathrm{HP}, N}(t)-P_{\mathrm{MT}}(t)+P_{\mathrm{MT}, N}(t) \\
& =P_{\mathrm{SC}}(t)+\Delta P_{\mathrm{HP}}(t)-\Delta P_{\mathrm{MT}}(t),
\end{aligned}
$$

where $P_{\text {tie-lineo }}(t)$ is the target power of tie-line at time $t$; $\Delta P_{\mathrm{HP}}(t)$ and $\Delta P_{\mathrm{MT}}(t)$ represent the power component of the heat pumps and the micro gas turbines participating in smoothing the power fluctuations of the tie-line at time $t$, respectively; and $P_{\mathrm{HP}, N}(t)$ and $P_{\mathrm{MT}, N}(t)$ are the rated operating power of heat pumps and micro gas turbines at time $t$, respectively.

Supercapacitors have the fastest power response speed and can bear high-frequency components of power fluctuations. For the heating equipment in the cogeneration system, the heat pumps and micro gas turbines have a capacity margin for smoothing power: the heat pumps have the slowest power response speed and can bear the low-frequency components in the power fluctuations by regulating the compressor output [11]. The power response speed of the micro gas turbines is between the supercapacitors and the heat pumps, and therefore the micro gas turbines can undertake the intermediate-frequency components of power fluctuations by regulating the injection power of natural gas.

Firstly, according to the different power response speeds of heat pumps, micro gas turbines, and supercapacitors, the corresponding low-pass filtering time constant is determined and the preoutput components of heat pumps, micro gas turbines, and supercapacitors can be obtained, respectively. Secondly, considering the output constraints of heat pumps and micro gas turbines, the SOC of supercapacitors, and virtual energy storage state, the equipment state mapping set is introduced to carry out real-time equipment correction preoutput.

\subsection{Preoutput Calculation Based on Low-Pass Filtering.} The power fluctuations of the tie-line are firstly low-pass filtered to obtain the preoutput of the heat pump, which is the low-frequency component. Then, the remaining medium-high frequency power fluctuations are filtered similarly, and the preoutput of micro gas turbines and supercapacitors can be determined. The expressions of the preoutput of the heat pumps, micro gas turbines and supercapacitors are shown in the following equations:

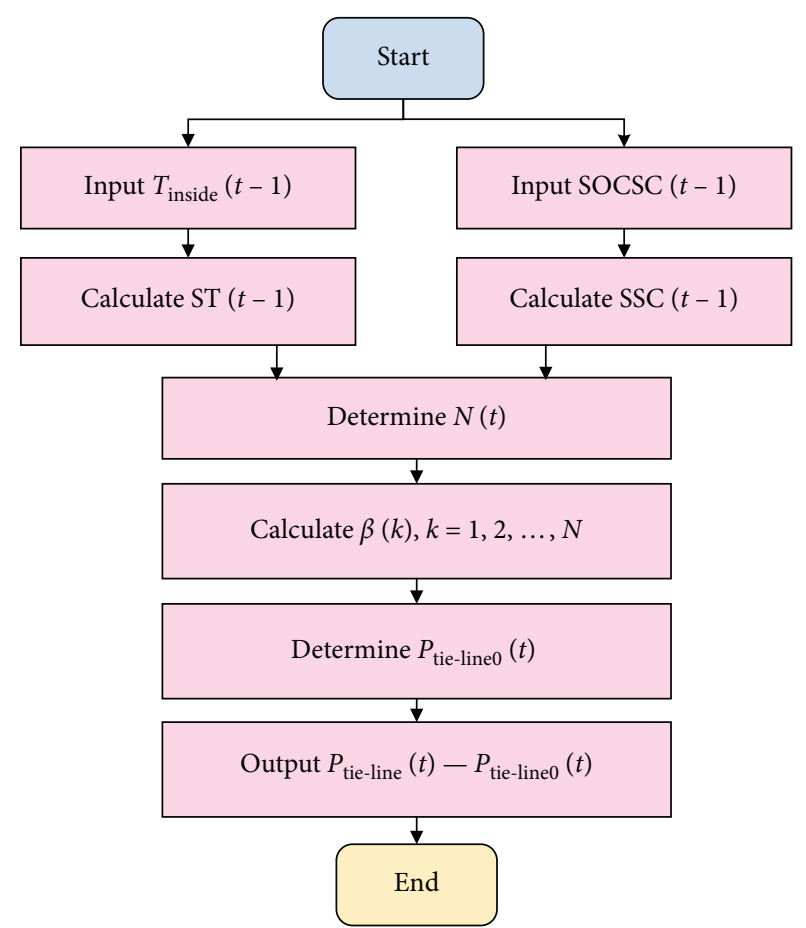

FIGURE 2: Flowchart of target power smoothing strategy.

$$
\begin{aligned}
\Delta P_{\mathrm{HP} \_ \text {ref }}(t)= & \frac{\lambda_{\mathrm{HP}}}{\lambda_{\mathrm{HP}}+\Delta t} \times \Delta P_{\mathrm{HP} \_ \text {ref }}(t-1) \\
& +\frac{\Delta t}{\lambda_{\mathrm{HP}}+\Delta t} \times\left[P_{\text {tie-line }}(t)-P_{\text {tie-line0 }}(t)\right],
\end{aligned}
$$

$$
\begin{aligned}
\Delta P_{\mathrm{MT} \_ \text {ref }}(t)= & \frac{\lambda_{\mathrm{MT}}}{\lambda_{\mathrm{MT}}+\Delta t} \times \Delta P_{\mathrm{MT} \_ \text {ref }}(t-1) \\
& +\frac{\Delta t}{\lambda_{\mathrm{MT}}+\Delta t} \\
& \times\left[P_{\text {tie-line } 0}(t)-P_{\text {tie-line }}(t)+\Delta P_{\mathrm{HP} \_ \text {ref }}(t)\right],
\end{aligned}
$$

$$
\begin{aligned}
P_{\mathrm{SC} \_ \text {ref }}(t)= & P_{\text {tie-line }}(t)-P_{\text {tie-line0 }}(t)-\Delta P_{\text {HP_ref }}(t) \\
& +\Delta P_{\text {MT_ref }}(t),
\end{aligned}
$$

where $\Delta P_{\text {HP_ref, }} \Delta P_{\text {MT_ref, }}$, and $P_{\text {SC_ref }}$ are the preoutput of the heat pumps, micro gas turbines, and supercapacitors, respectively. $\lambda_{\mathrm{HP}}$ and $\lambda_{\mathrm{MT}}$ are the filtering time constant of heat pumps and micro gas turbines, respectively. $\Delta t$ is defined as the system sampling interval.

\subsection{Preoutput Correction Based on State Mapping Set.} The above preoutput calculation is based on the power response characteristics of heat pumps, micro gas turbines, and supercapacitors, without considering the virtual energy storage state and the SOC of supercapacitors. The equipment state mapping set and correction factor are introduced to modify the preoutput. 
Input parameters of the heat pump state mapping set, the gas turbine state mapping set, and the supercapacitor state mapping set are shown in the following equations, respectively, and their values are per unit values:

$$
\begin{aligned}
x_{\mathrm{HP}, 1}(t) & =\frac{\Delta P_{\mathrm{HP}}(t-1)}{P_{\mathrm{HP}, N}}, \\
x_{\mathrm{HP}, 2}(t) & =x_{\mathrm{MT}, 1}(t)=S_{\mathrm{T}}(t-1) \\
& =\frac{T_{\text {inside }}(t-1)-T_{\text {inside } 0}}{\left(T_{\text {inside_max }}-T_{\text {inside_min }}\right) / 2}, \\
x_{\mathrm{MT}, 2}(t) & =\frac{\Delta P_{\mathrm{MT}}(t-1)}{P_{\mathrm{MT}, N}}, \\
x_{\mathrm{SC}, 1}(t) & =S_{\mathrm{SC}}(t-1) \\
& =\frac{\mathrm{SOC}_{\mathrm{SC}}(t-1)-\mathrm{SOC}_{\mathrm{SC} 0}}{\left(\mathrm{SOC}_{\mathrm{SC} \_ \text {max }}-\mathrm{SOC}_{\mathrm{SC} \_ \text {min }}\right) / 2}, \\
x_{\mathrm{SC}, 2}(t) & =P_{\text {tie-line }}(t)-P_{\text {tie-line }}(t-1) .
\end{aligned}
$$

The heat pumps preoutput correction is based on the heat pumps' real-time running state and virtual energy storage state index. When the power fluctuations of the tieline are positive, if $x_{\mathrm{HP}, 1}(t)$ is high, it indicates that the compressor's output is weak to increase and strong to decrease, so the correction factor should be reduced to avoid severe overload of the heat pumps; if $x_{\mathrm{HP}, 2}(t)$ is close to 1 , that is, the room temperature is close to the allowed upper limit, at this time the compressor output should be cut down and the heat power of the heat pumps should be reduced to avoid room temperature exceeding the allowed upper limit. When the power fluctuations of the tie-line are negative, if $x_{\mathrm{HP}, 1}(t)$ is low, the compressor output reduction capacity is very weak, and the correction factor should be increased to avoid a light load of the heat pumps; and if the room temperature is close to the lower limit, the compressor output should be increased and the heat power of heat pumps should be increased to avoid room temperature exceeding the lower limit.

The micro gas turbines preoutput correction is based on the virtual energy storage state index and natural gas injection power state. When the power fluctuations are positive, if $x_{\mathrm{MT}, 2}(t)$ is high and the micro gas turbines have a strong lowering capacity, it is necessary to reduce the correction factor to avoid excessive natural gas injection power and if the virtual energy storage state index $x_{\mathrm{MT}, 1}(t)$ is close to 1 , the heating power of the micro gas turbines should be reduced to avoid room temperature exceeding the allowed upper limit. The analysis of negative power fluctuations of the tie-line is similar.

The preoutput correction of the supercapacitors is based on the SOC and the power fluctuations of the tie-line. When the power fluctuations are positive, if $x_{\mathrm{SC}, 1}(t)$ is high, the correction factor should be reduced accordingly to reduce the supercapacitors' output, so as to avoid the SOC exceeding the allowed upper limit. Considering the supercapacitors' fast power response speed, if the positive fluctuation rate of the tie-line $x_{\mathrm{SC}, 2}(t)$ is large, the correction factor should be increased correspondingly to increase the output of the supercapacitors. The analysis of negative power fluctuations of the tie-line is similar.

Due to the space limit, this paper only presents the heat pump state mapping set when the power fluctuations of the tie-line are positive, as shown in Table 1. Input parameters $x_{\mathrm{HP}, 1}(t)$ are divided into 5 state intervals, [-0.1, -0.05$)$, $[-0.05,-0.02),[-0.02,0.02],[-0.02,0.02],(0.02,0.05],(0.05$, $0.1]$, and $x_{\mathrm{HP}, 2}(t)$ are divided into 3 state intervals, $[-1,-0.5)$, $[-0.5,0.5],(0.5,1]$, and can therefore map to 15 states. $\mu_{\mathrm{HP}}(t)$ is defined as the correction factor and $\Delta \mu_{\mathrm{HP}}(t)$ is the variation of the correction factor of heat pumps at time $t$ compared with that at time $t-1$. Each state corresponds to a specific value of $\Delta \mu_{\mathrm{HP}}(t)$, of which the set of values are $\left\{-3 \mu_{0}\right.$, $\left.-2 \mu_{0},-\mu_{0}, 0, \mu_{0}, 2 \mu_{0}, 3 \mu_{0}\right\}$, and $\mu_{0}$ represents the unit basis of the correction factor and is a fixed constant. The expressions of $\mu_{\mathrm{HP}}(t)$ and $\Delta P_{\mathrm{HP}}(t)$ are shown in the following equations, respectively:

$$
\begin{aligned}
\mu_{\mathrm{HP}}(t) & =\mu_{\mathrm{HP}}(t-1)+\Delta \mu_{\mathrm{HP}}(t), \\
\Delta P_{\mathrm{HP}}(t) & =\left[1+\mu_{\mathrm{HP}}(t)\right] \times \Delta P_{\mathrm{HP} \_r e f}(t) .
\end{aligned}
$$

Similar to equation (22), the expressions of the corrected output component of micro gas turbines and supercapacitors are shown in the following equations, respectively:

$$
\begin{gathered}
\Delta P_{\mathrm{MT}}(t)=\left[1+\mu_{\mathrm{MT}}(t)\right] \times \Delta P_{\mathrm{MT} \_r e f}(t), \\
P_{\mathrm{SC}}(t)=\left[1+\mu_{\mathrm{SC}}(t)\right] \times P_{\text {SC_ref }}(t) .
\end{gathered}
$$

To sum up, the smoothing community microgrid tie-line power procedure considering the virtual energy storage technology is shown in Figure 3. The target power of the tieline can be determined by the weighted sliding average filtering algorithm. The preoutput components of heat pumps, micro gas turbines, and supercapacitors can be obtained through low-pass filtering, respectively. Considering the constraints of the equipment and the virtual energy storage state's running state, the correction factor is introduced to determine the final output based on the equipment state mapping set.

\section{Case Study}

The topology structure of the community microgrid is shown in Figure 4. The capacity of supercapacitors is $50 \mathrm{kWh}$, and the charging/discharging limit is $50 \mathrm{~kW}$. The capacity of heat pumps and micro gas turbines is $50 \mathrm{~kW}$ and $100 \mathrm{~kW}$, respectively. The target reference value of room temperature is $22^{\circ} \mathrm{C}$. The daily power curve of microgrid equipment and environment temperature curve are displayed in Figures 5 and 6, respectively.

The power fluctuations smoothing results of the community microgrid are depicted in Figure 7 (a). The comparison of target power output before and after smoothing is shown in Table 2. Referring to [31, 32], the day-ahead regulation reserve price is $\$ 15.68 / \mathrm{MWh}$, and the regulation 
TABLE 1: State mapping set of heat pumps $\left(P_{\text {tie-line }}(t) \geq 0\right)$.

\begin{tabular}{lccc}
\hline$x_{\mathrm{HP}, 1}$ & \multicolumn{3}{c}{$x_{\mathrm{HP}, 2}$} \\
& {$[-1,-0.5)$} & {$[-0.5,0.5]$} & $(0.5,1]$ \\
\hline$[-0.1,-0.05)$ & $3 \mu_{0}$ & $2 \mu_{0}$ & $\mu_{0}$ \\
{$[-0.05,-0.02)$} & $2 \mu_{0}$ & $\mu_{0}$ & 0 \\
{$[-0.02,0.02]$} & $\mu_{0}$ & $-\mu_{0}$ & $-\mu_{0}$ \\
$(0.02,0.05]$ & 0 & $-2 \mu_{0}$ & $-2 \mu_{0}$ \\
$(0.05,0.1]$ & $-\mu_{0}$ & $-3 \mu_{0}$ \\
\hline
\end{tabular}

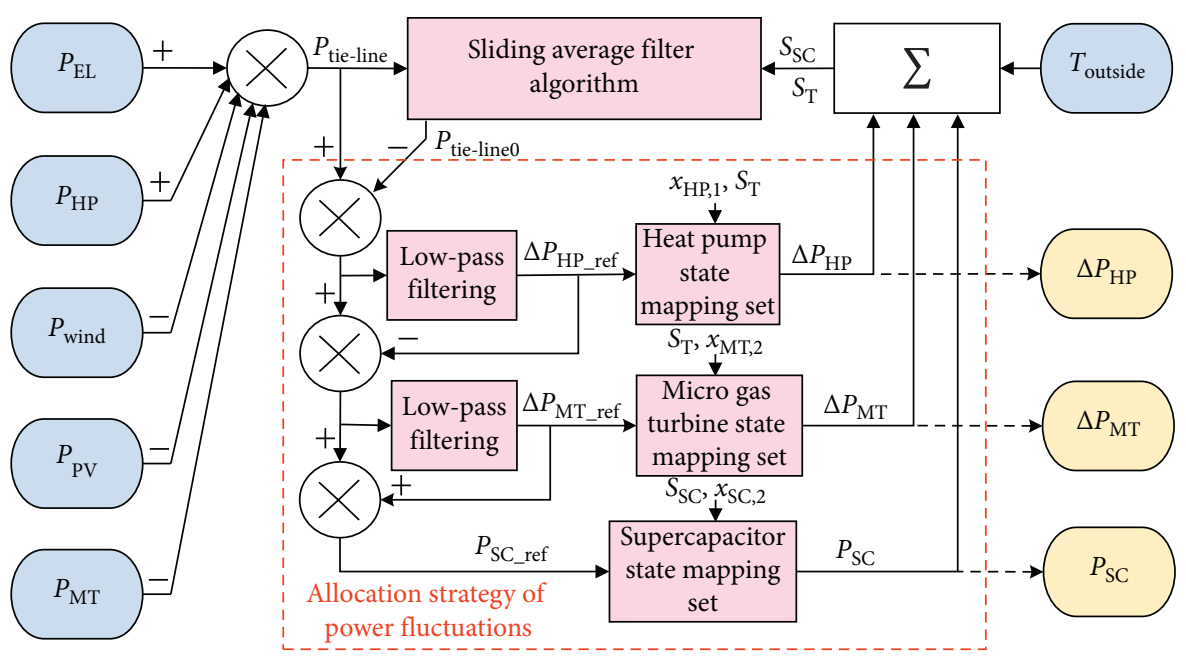

FIgURE 3: Structure of smoothing power fluctuations of the microgrid tie-line.

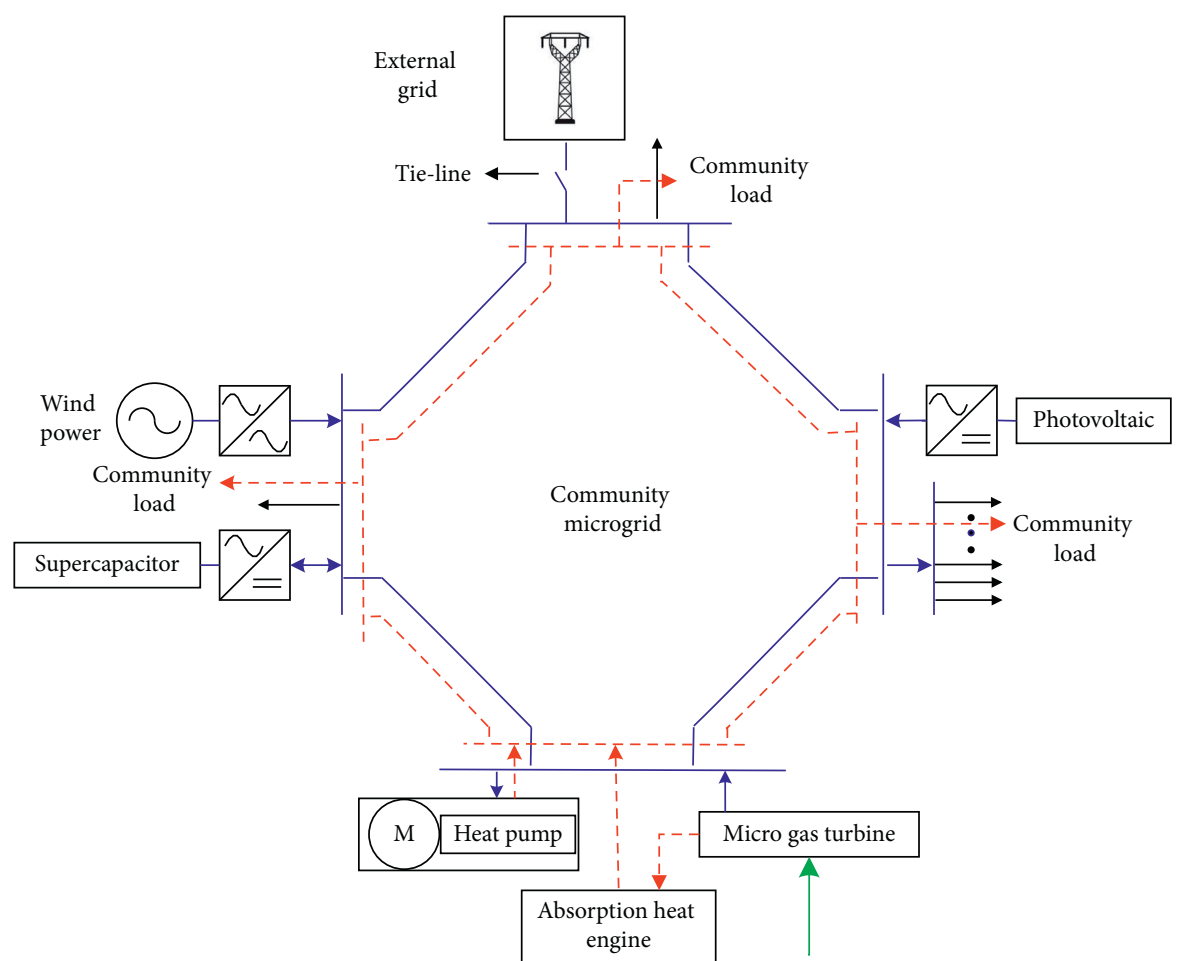

$\rightarrow$ Heat

Gas

$\longrightarrow$ Power

FIgURE 4: Topology of the community microgrid combined with heat and power system. 


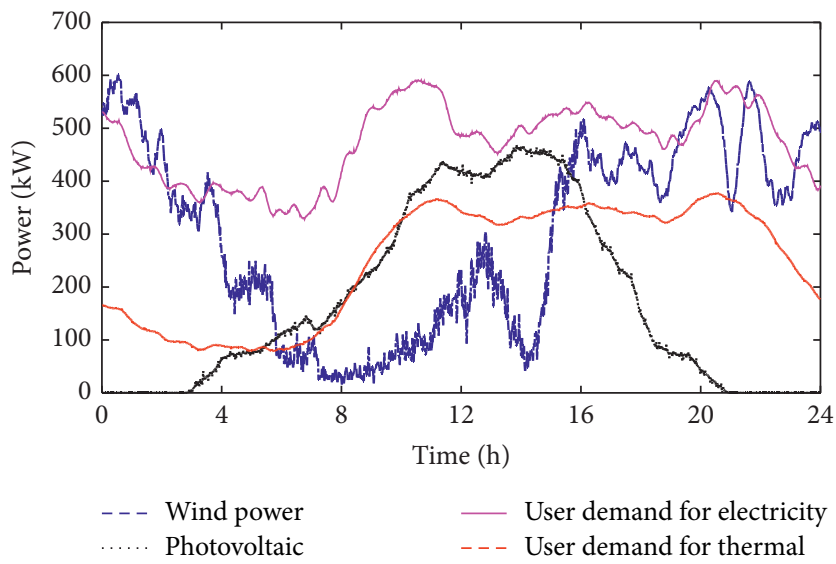

FIgURE 5: Daily power curves of the microgrid.

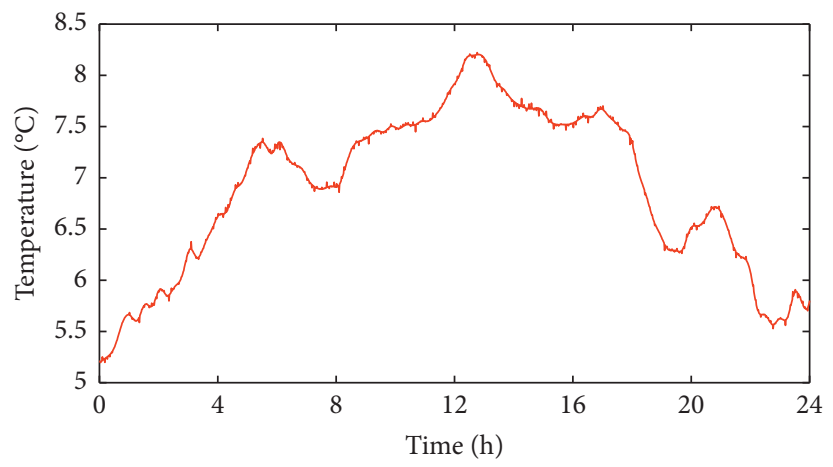

FIGURE 6: Temperature curve of the environment outside.
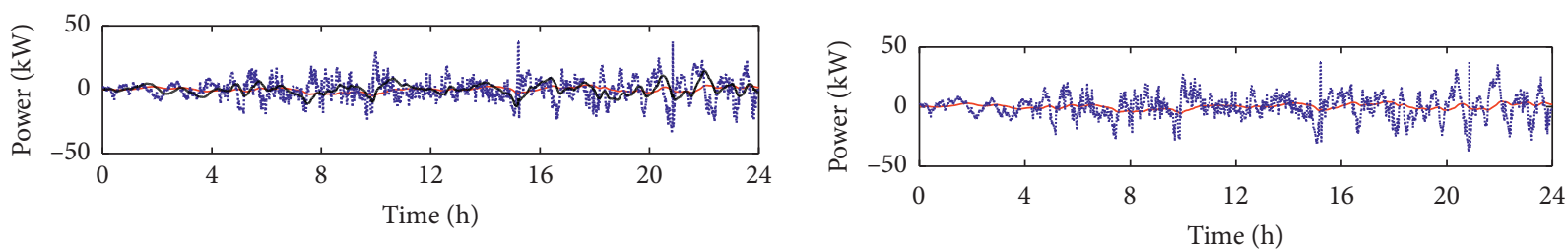

- Heat pumps

..... Supercapacitor

- - Micro gas turbines

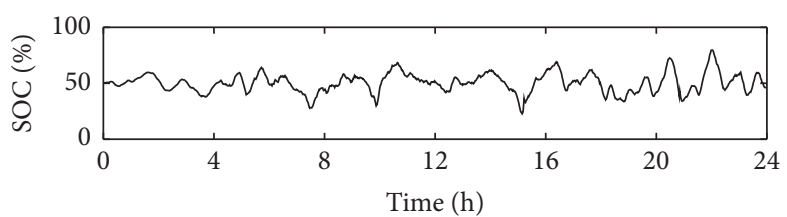

_- Heat pumps

Supercapacitor

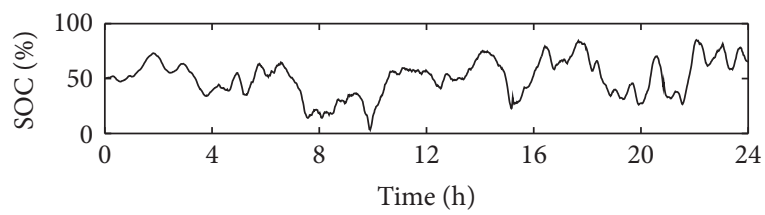

— Supercapacitor

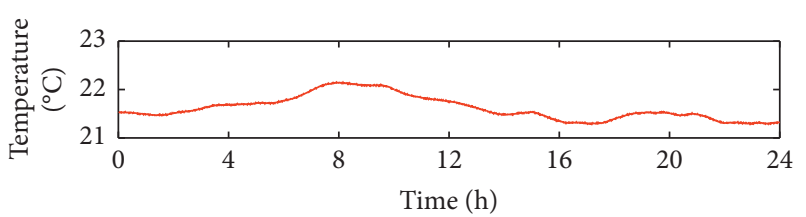

(a)

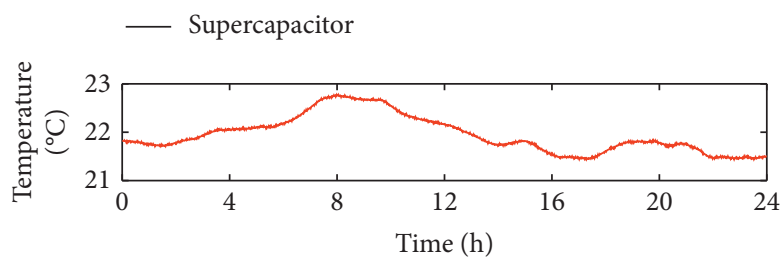

(b)

FIgURE 7: Power and SOC of microgrid equipment: (a) with micro gas turbine and (b) without micro gas turbine. 
TABLE 2: Target power output before/after smoothing.

\begin{tabular}{lccc}
\hline Parameter & $\begin{array}{c}\text { Maximum rate of power change } \\
(\mathrm{kW} / \mathrm{min})\end{array}$ & $\begin{array}{c}\text { Sum of power change rate } \\
(\mathrm{MW} / \mathrm{min})\end{array}$ & $\begin{array}{c}\text { Regulation reserve cost per year } \\
\text { (US dollar) }\end{array}$ \\
\hline Before & 121.93 & 7.83 & 746.87 \\
After & 8.34 & 1.83 & 174.55 \\
Percentage decline (\%) & 86.1 & 76.6 & 76.6 \\
\hline
\end{tabular}

reserve cost per year can be estimated, as shown in Table 2 . After applying the micro gas turbines, heat pumps, and supercapacitors, the microgrid power output characteristics is greatly improved. Compared with supercapacitors' output characteristics, the heat pumps and micro gas turbines have a slower response speed and are used to compensate for the low-frequency and middle-frequency component of target power fluctuation, respectively. The supercapacitors bear the high-frequency of power fluctuations after being smoothed by heat pumps and micro gas turbines.

Comparatively, the target power curve when the micro gas turbines do not participate is displayed in Figure 7(b). Under this condition, the heat pumps compensate the lowfrequency fluctuations component, while the supercapacitors undertake the high-frequency component. It can be found that the SOC of supercapacitors is very close to the allowable upper and lower limits of SOC. At this time, the supercapacitors' installed capacity should be increased correspondingly to improve the overall charging/discharging capacity of the cogeneration system to bear the target power fluctuation and extend the service life of the supercapacitors. In addition, by comparing the room temperature curves in Figures 7(a) and 7(b), it can be seen that the micro gas turbines can adjust the injection power of natural gas and correspondingly change the power to bear power fluctuations. Meanwhile, the effect of heat power on room temperature is opposite to that of heat pumps. Therefore, the range of room temperature variation on the user side is smaller and the temperature control effect is improved through the coordinated control of heat pumps and micro gas turbines.

The power smoothing effect of the community microgrid tie-line is shown in Figure 8. Based on the sliding average filtering algorithm, the target power of the tie-line is determined, and the preoutput of the micro gas turbine and heat pump is modified according to the state mapping table. It can be found from Figure 8 that the power smoothing effect of the tie-line is significantly improved with the cooperation of supercapacitors, heat pumps, and micro gas turbines. The more consistent the actual power of the tie-line is with the target power, the less reserve capacity the system needs and the better the economic benefit of the community microgrid is. The case study verifies the correctness and effectiveness of the power smoothing method

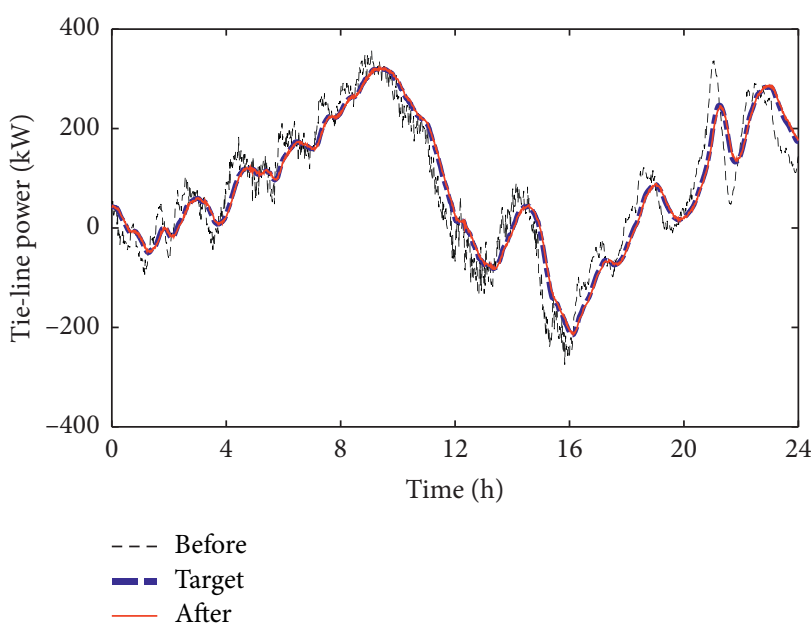

Figure 8: Smoothing effect of the tie-line power.

for community microgrid tie-line with virtual energy storage.

\section{Conclusions}

In this paper, a virtual energy storage model is established considering the heat storage capacity of buildings on the user side of the community microgrid, and a power smoothing method of community microgrid tie-line for cogeneration system is proposed. This method is no longer limited to single heating equipment, and coordinated control of heat pumps and micro gas turbines can smooth the power fluctuations more effectively and ensure the user side room temperature in a reasonable range. Coordination between the virtual energy storage and supercapacitors can achieve deep coupling between electric energy and thermal energy and reduce electric energy storage equipment's capacity cost. The coordination of heat pumps, micro gas turbines, and supercapacitors can significantly improve the energy control flexibility of microgrid and the comprehensive utilization efficiency of electricity, heat, and other energy.

\section{Data Availability}

The data used to support the study are available within the article. 


\section{Conflicts of Interest}

The authors declare no conflicts of interest.

\section{Acknowledgments}

This work was supported by the World Class Urban Distribution Network Demonstration Project of Suzhou Historic District. This research was funded by State Grid Suzhou Power Supply Company.

\section{References}

[1] L. Igualada, C. Corchero, M. Cruz-Zambrano, and F.-J. Heredia, "Optimal energy management for a residential microgrid including a vehicle-to-grid system," IEEE Transactions on Smart Grid, vol. 5, no. 4, pp. 2163-2172, 2014.

[2] C. Feng, W. Liu, F. Wen, Z. Li, M. Shahidehpour, and X. Shen, "Expansion planning for active distribution networks considering deployment of smart management technologies," IET Generation, Transmission \& Distribution, vol. 12, no. 20, pp. 4605-4614, 2018.

[3] L. Che, M. Shahidehpour, A. Alabdulwahab, and Y. Al-Turki, "Hierarchical coordination of a community microgrid with AC and DC microgrids," IEEE Transactions on Smart Grid, vol. 6, no. 6, pp. 3042-3051, 2015.

[4] X. Chen, C. Kang, M. O’Malley et al., "Increasing the flexibility of combined heat and power for wind power integration in China: modeling and implications," IEEE Transactions on Power Systems, vol. 30, no. 4, pp. 1848-1857, 2014.

[5] C. Feng, Z. Li, M. Shahidehpour, F. Wen, and Q. Li, "Stackelberg game based transactive pricing for optimal demand response in power distribution systems," International Journal of Electrical Power \& Energy Systems, vol. 118, 2020.

[6] P. Shamsi, H. Xie, A. Longe et al., "Economic dispatch for an agent-based community microgrid," IEEE Transactions on Smart Grid, vol. 7, no. 5, pp. 2317-2324, 2015.

[7] C. Feng, Z. Li, M. Shahidehpour, F. Wen, W. Liu, and $\mathrm{X}$. Wang, "Decentralized short-term voltage control in active power distribution systems," IEEE Transactions on Smart Grid, vol. 9, no. 5, pp. 4566-4576, 2017.

[8] P. Tian, X. Xiao, K. Wang et al., "A hierarchical energy management system based on hierarchical optimization for microgrid community economic operation," IEEE Transactions on Smart Grid, vol. 7, no. 5, pp. 2230-2241, 2015.

[9] K. Rahbar, C. C. Chai, and R. Zhang, "Energy cooperation optimization in microgrids with renewable energy integration," IEEE Transactions on Smart Grid, vol. 9, pp. 1482-1493, 2016.

[10] L. Wang, R. Yan, F. Bai, T. K. Saha, and K. Wang, "A distributed inter-phase coordination algorithm for voltage control with unbalanced PV integration in LV systems," IEEE Transactions on Sustainable Energy, vol. 11, no. 4, pp. 26872697, 2020.

[11] L. Wang, R. Yan, and T. K. Saha, "Voltage regulation challenges with unbalanced PV integration in low voltage distribution systems and the corresponding solution," Applied Energy, vol. 256, 2019.

[12] K. Wang, X. Feng, J. B. Pang, J. Ren, C. X. Duan, and L. W. Li, "State of charge (SOC) estimation of lithium-ion battery based on adaptive square root unscented kalman filter," International Journal of Electrochemical Science, vol. 15, no. 9, pp. 9499-9516, 2020.
[13] G.-T. Xia, C. Li, K. Wang, and L.-W. Li, "Structural design and electrochemical performance of PANI/CNTs and $\mathrm{MnO} 2 /$ CNTs supercapacitor," Science of Advanced Materials, vol. 11, no. 8, pp. 1079-1086, 2019.

[14] K. Wang, L. Li, T. Zhang, and Z. Liu, "Nitrogen-doped graphene for supercapacitor with long-term electrochemical stability," Energy, vol. 70, pp. 612-617, 2014.

[15] Y. Zhou, Y. Huang, J. Pang, and K. Wang, "Remaining useful life prediction for supercapacitor based on long short-term memory neural network," Journal of Power Sources, vol. 440, 2019.

[16] H. A. Aalami and S. Nojavan, "Energy storage system and demand response program effects on stochastic energy procurement of large consumers considering renewable generation," IET Generation, Transmission \& Distribution, vol. 10, no. 1, pp. 107-114, 2016.

[17] Y. Zhou, Y. Wang, K. Wang et al., "Hybrid genetic algorithm method for efficient and robust evaluation of remaining useful life of supercapacitors," Applied Energy, vol. 260, pp. 114-169, 2020.

[18] F. Zhang, K. Meng, Z. Xu et al., "Battery ESS planning for wind smoothing via variable-interval reference modulation and self-adaptive SOC control strategy," IEEE Transactions on Sustainable Energy, vol. 8, pp. 695-707, 2016.

[19] Q. Wei, G. Shi, R. Song, and Y. Liu, "Adaptive dynamic programming-based optimal control scheme for energy storage systems with solar renewable energy," IEEE Transactions on Industrial Electronics, vol. 64, no. 7, pp. 5468-5478, 2017.

[20] J. Xiao, P. Wang, and L. Setyawan, "Hierarchical control of hybrid energy storage system in DC microgrids," IEEE Transactions on Industrial Electronics, vol. 62, no. 8, pp. 4915-4924, 2015.

[21] M. Zidar, P. S. Georgilakis, N. D. Hatziargyriou, T. Capuder, and D. Š.nd e, "Review of energy storage allocation in power distribution networks: applications, methods and future research," IET Generation, Transmission \& Distribution, vol. 10, no. 3, pp. 645-652, 2016.

[22] C. Bu, F. Li, K. Yin, J. Pang, L. Wang, and K. Wang, "Research progress and prospect of triboelectric nanogenerators as selfpowered human body sensors," ACS Applied Electronic Materials, vol. 2, no. 4, pp. 863-878, 2020.

[23] S. Kawachi, H. Hagiwara, J. Baba, K. Furukawa, E. Shimoda, and S. Numata, "Study on compensation of short term power fluctuation by use of heat pump air conditioning system based on real machine," IEEJ Transactions on Power and Energy, vol. 132, no. 1, pp. 77-85, 2012.

[24] P. S. Sauter, B. V. Solanki, C. A. Cañizares, K. Bhattacharya, and S. Hohmann, "Electric thermal storage system impact on northern communities' microgrids," IEEE Transactions on Smart Grid, vol. 10, no. 1, pp. 852-863, 2019.

[25] R. Wang, D. Wang, H. J. Jia et al., "A coordination control strategy of battery and virtual energy storage to smooth the micro-grid tie-line power fluctuations," Proceedings of the CSEE, vol. 20, pp. 5124-5134, 2015.

[26] L. Xu, G. Y. Yang, Z. Xu et al., "Combined scheduling of electricity and heat in a microgrid with volatile wind power," Automation of Electric Power Systems, vol. 35, no. 9, pp. 53-60, 2011.

[27] X. Jin, Y. Mu, H. Jia, J. Wu, T. Jiang, and X. Yu, “Dynamic economic dispatch of a hybrid energy microgrid considering building based virtual energy storage system," Applied Energy, vol. 194, pp. 386-398, 2017. 
[28] X. Feng, Y. Zhang, L. Kang et al., "Integrated energy storage system based on triboelectric nanogenerator in electronic devices," Frontiers of Chemical Science and Engineering, pp. 1-13, 2020.

[29] C. Cristofari, R. Norvaišienè, J. L. Canaletti, and G. Notton, "Innovative alternative solar thermal solutions for housing in conservation-area sites listed as national heritage assets," Energy and Buildings, vol. 89, pp. 123-131, 2015.

[30] A. Buonomano and A. Palombo, "Building energy performance analysis by an in-house developed dynamic simulation code: an investigation for different case studies," Applied Energy, vol. 113, pp. 788-807, 2014.

[31] L. Rodríguez-Guerrero, O. Santos-Sánchez, and S. Mondié, "A constructive approach for an optimal control applied to a class of nonlinear time delay systems," Journal of Process Control, vol. 40, pp. 35-49, 2016.

[32] L. Lu, F. Wen, Y. Xue, and J. Kang, "Economic analysis of ancillary service provision by plug-in electric vehicles," $A u$ tomation of Electric Power Systems, vol. 37, pp. 43-49, 2013. 